\title{
Cytomegalovirus retinitis and HIV: Case reviews from KwaZulu-Natal Province, South Africa
}

\author{
R Hassan-Moosa, ${ }^{1}$ MB BCh, MPH; T Chinappa, ${ }^{1}$ LRCP LRCS LM (Ireland); L Jeena, ${ }^{1}$ 6th year medical student; \\ L Visser, ${ }^{3} \mathrm{MB}$ ChB, FCOphth (SA); K Naidoo, ${ }^{1,2} \mathrm{MB} \mathrm{ChB}, \mathrm{PhD}$ \\ ${ }^{1}$ Centre for the AIDS Programme of Research in South Africa (CAPRISA), Doris Duke Medical Research Institute, University of KwaZulu-Natal, \\ Durban, South Africa \\ ${ }^{2}$ MRC-CAPRISA HIV-TB Pathogenesis and Treatment Research Unit, Doris Duke Medical Research Institute, University of KwaZulu-Natal, \\ Durban, South Africa \\ ${ }^{3}$ Department of Ophthalmology, Nelson Mandela Medical School of Medicine, University of KwaZulu-Natal, Durban, South Africa
}

Corresponding author: R Hassan-Moosa (razia.hassan-moosa@caprisa.org)

\begin{abstract}
Background. Retinal cytomegalovirus (CMV) infection is a common opportunistic infection and remains a significant contributor to visual loss in patients with AIDS. We highlight the poor outcomes of CMV retinitis in three HIV-infected patients who were initiated on antiretroviral therapy (ART). We conducted a retrospective chart review of advanced stage HIV-infected patients with known CMV retinitis. Case 1. A 37-year-old man, with a CD4+ cell count of $35 \mathrm{cells} / \mu \mathrm{L}$, presented for ART initiation with a 5-month history of visual loss in his left eye. Fundoscopy showed left eye CMV retinitis and right eye HIV retinopathy. ART and 5 months of weekly intravitreal ganciclovir injections (left eye) were commenced. Six-month outcomes included virological suppression, and visual acuity in the right eye of $6 / 6$ and in the left eye of $3 / 60$.

Case 2. A 31-year-old woman, with a CD4+ cell count of 39 cells $/ \mu \mathrm{L}$ and on tuberculosis therapy, presented for ART initiation. She presented with a 2-month history of decreased visual acuity. Fundoscopy showed bilateral CMV retinitis, which was more pronounced in the left eye. ART and 8 months of intravitreal ganciclovir injections were commenced. Six-month outcomes included virological suppression and visual acuity in the right eye of $6 / 9$, and in the left eye of $6 / 24$.

Case 3. A 29-year-old woman, with a CD4+ cell count of 24 cells/ $\mu \mathrm{L}$, who was on tuberculosis therapy and ART, complained of blurred vision at her 2-month ART follow-up visit. Fundoscopy showed bilateral retinal detachment secondary to CMV retinitis. While silicone oil tamponade and subsequent retinectomy successfully repaired the right eye, extensive damage rendered the left eye irreparable. Six-month outcomes included virological suppression, with 6/120 visual acuity in the right eye and complete blindness in the left eye.

Conclusion. CMV retinitis causes debilitating, permanent sequelae, which is preventable by ART initiation at higher CD4+ cell counts. Despite achieving virological suppression, vision could not be completely restored in these patients, irrespective of the severity of CMV retinitis.
\end{abstract}

S Afr Med J 2017;107(10):843-846. DOI:10.7196/SAMJ.2017.v107i10.12740

Cytomegalovirus (CMV) is a common opportunistic infection and a significant contributor to visual loss in patients with advanced stage HIV. ${ }^{[1]}$ CMV seropositivity in Africa, specifically in subSaharan Africa, is highly endemic with reported seropositivity rates approaching $100 \% \cdot{ }^{[2]}$ Despite antiretroviral therapy (ART), CMV infection in AIDS patients remains a significant risk factor for end organ disease and AIDS-related mortality. ${ }^{[3,4]}$ Prior to the introduction of ART) 30 - 40\% of HIV-infected individuals developed CMV retinitis. ${ }^{[5]}$ However, the introduction of ART has resulted in a precipitous decline in CMV retinitis infections, which is particularly evident in the developed world. ${ }^{[6]}$ Reported prevalence rates of CMV retinitis in HIV-infected patients varied between $<5 \%$ in South Africa (SA) to $>30 \%$ in South East Asia, ${ }^{[7]}$ with a study in Cape Town reporting a prevalence rate of $0.8 \%(p<0.001)$ in patients with $\mathrm{CD} 4+$ cell counts $<200 \mu / \mathrm{L}{ }^{[8]}$ Data on local incidence rates are limited with the literature reporting sub-Saharan African incidence rates of $<10 \% .{ }^{[9]}$

Resource-limited settings are challenged by inaccessibility of ocular care for HIV-infected patients on ART programmes, owing to a lack of skilled staff to conduct ophthalmic examinations. The mainstay of diagnosis of CMV retinitis is fundoscopy through a dilated pupil. ${ }^{[10]}$ However, as CMV retinitis is asymptomatic in almost half of affected patients in the early stages of disease, ${ }^{[11]}$ routine screening fundoscopy for those at high risk of CMV disease by a trained clinician, not necessarily an ophthalmologist, is necessary. A review of the data from a 3-year pilot programme in Myanmar ${ }^{[12]}$ showed the feasibility of CMV retinitis management at primary care level in resource-poor settings. Like other HIV-associated major opportunistic infections, CMV retinitis was diagnosed and treated by AIDS clinicians (non-ophthalmologists), with the appropriate training and support, prompting calls for the WHO to include routine ophthalmoscopy for all patients presenting for ART initiation with low CD4+ cell counts $(<100$ cells $/ \mu \mathrm{L})$ in AIDS treatment guidelines. ${ }^{[4,12]}$

CMV retinitis is a slow and progressive disease in patients with advanced stage HIV. ${ }^{[2]}$ Initial symptoms include visual field defects, the visualisation of floaters, flashes and a decline in vision, which initially occurs in one eye. ${ }^{[13]}$ The disease is diagnosed presumptively by means of fundoscopy, unlike systemic CMV disease, which requires confirmation by biopsy. ${ }^{[11]}$ Characteristic findings on fundoscopy include dense retinal whitening, haemorrhage, and a typical 'brushfire' retinitis pattern that distributes along blood vessels with small white satellite lesions at the border. ${ }^{[2]} \mathrm{CMV}$ retinitis is commonly confused with HIV retinopathy; however, the latter fades and condenses over time while CMV retinitis progresses. ${ }^{[2]}$ When left untreated, CMV retinitis can progress to retinal detachment with 
direct damage to the macula or optic nerve, resulting in permanent blindness. ${ }^{[2]}$ Blindness caused by CMV retinitis is irreversible and can occur prior to complete retinal destruction, which was responsible for $>90 \%$ of HIV-associated blindness in the pre-ART era. ${ }^{[2]}$

A total of 1815 patients were enrolled into the PEPFAR-funded CAPRISA AIDS Treatment Programme (CAT) at the eThekwini site in Durban, South Africa (SA) between June 2004 and August 2013. In this report, we highlight the three patients in this cohort who developed CMV retinitis.

Approval for data collection and analysis for the CAT programme was obtained from the University of KwaZulu-Natal Biomedical Research Ethics Committee (ref. no. E248/05).

\section{Case reports}

\section{Case 1}

In June 2011, a 37-year-old man presented for ART initiation with a baseline CD4+ cell count of 35 cells/ $\mu \mathrm{L}$. He complained of a 5-month history of painless visual loss in the left eye. ART was commenced and he was referred to the ophthalmology clinic. Fundoscopy of the right eye showed a clear cornea, normal lens, a pink disc and cotton wool spots (Fig. 1), with a baseline visual acuity of $6 / 18$. Poorer visual acuity of 3/60 was evident in the left eye, with fundoscopic examination showing dense retinal whitening and full-thickness necrosis (Fig. 2).

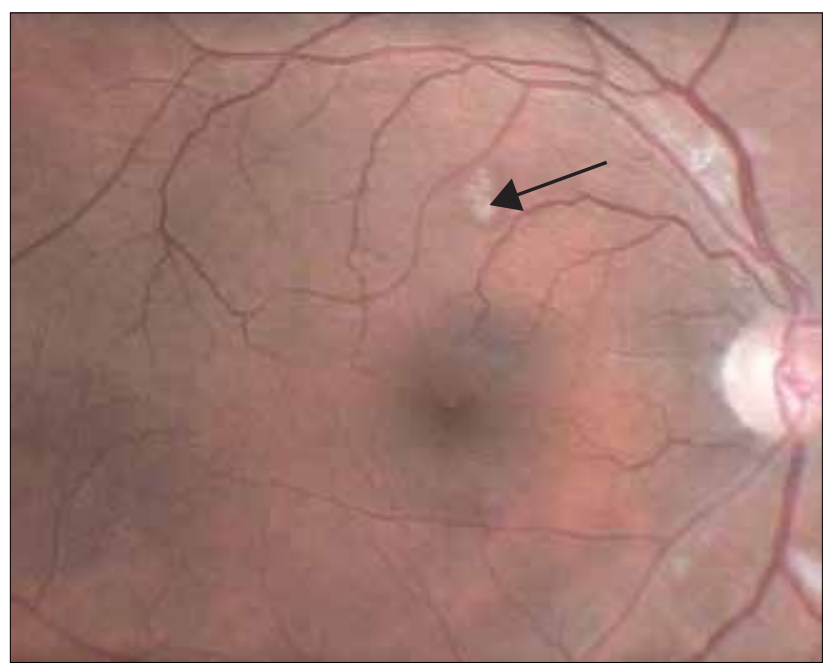

Fig. 1. Fundoscopy of the right eye (case 1) showing a clear cornea, normal lens, a pink disc and cotton spots.

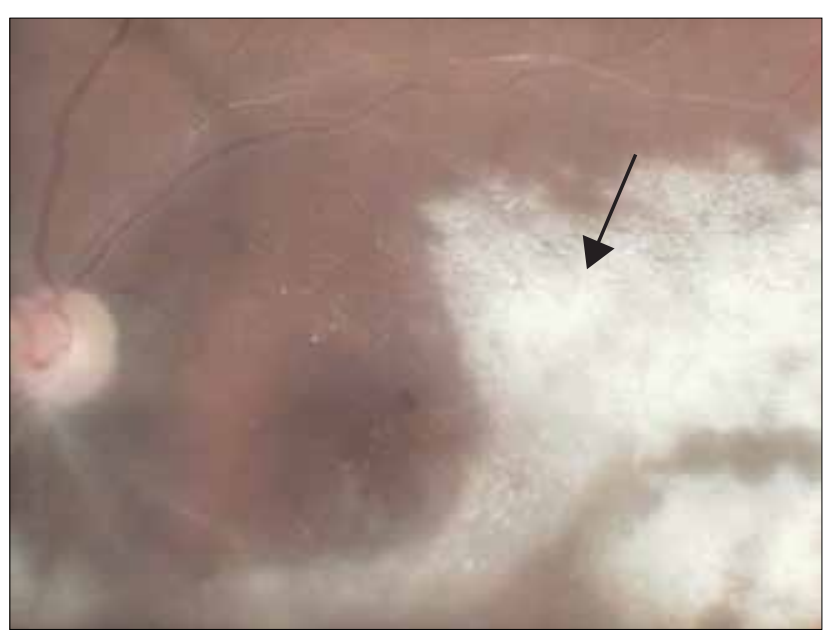

Fig. 2. Left eye fundoscopy (case 1) showing dense retinal whitening and fullthickness necrosis.
A diagnosis of CMV retinitis in the left eye and HIV retinopathy in the right eye was concluded. Weekly intravitreal ganciclovir injections into the left eye were commenced, and maintained for a total of 6 months. At 6 months post ART initiation, the patient's CD4+ cell count was 63 cells $/ \mu \mathrm{L}$ and his viral load was 42 copies $/ \mathrm{mL}$. His last ophthalmology report showed an improvement in visual acuity to $6 / 6$ with no other pathology in the right eye. No visual improvement was noted in the left eye; visual acuity remained static at $3 / 60$. The patient remained clinically stable otherwise.

\section{Case 2}

A 31-year-old woman, who was on TB treatment, presented for ART initiation in June 2007 with a baseline CD4+ cell count of 39 cells $/ \mu \mathrm{L}$. She complained of a 2-month history of deteriorating vision in both eyes, with the left eye being worse than the right. She was referred to the ophthalmology clinic and fundoscopy showed pigmented keratic precipitates on the cornea and frosted branch angiitis in the left eye. Retinitis, angiitis and hyperaemic discs were observed in the right eye. Bilateral CMV retinitis was confirmed. The patient was initiated on intravitreal ganciclovir injections in both eyes, and she started ART 6 weeks later. She received ganciclovir injections for 8 months and the visual acuity in her right eye improved to 6/9, and in her left eye to $6 / 24$. The patient maintained viral suppression and had increased CD4+ cell counts of 220 and 440 cells $/ \mu \mathrm{L}$ at 6 and 18 months, respectively.

\section{Case 3}

A 29-year-old woman, who was on TB treatment, presented for ART initiation in October 2006 with a baseline CD4+ cell count of 24 cells $/ \mu \mathrm{L}$. The patient complained of blurred vision at her 2-month ART follow-up visit, and was referred to the ophthalmology clinic. Decreased visual acuity and impaired peripheral vision with a bilateral hemianopia was noted. Furthermore, bilateral retinal detachment secondary to CMV retinitis was observed on fundoscopy. The right eye was repaired with silicone oil tamponade. However, owing to poor prognosis secondary to extensive damage from complete retinal detachment in the left eye, surgical repair was not an option. Retinectomy and a repeat silicone oil tamponade was performed on the right eye ten months later. She was blind in the left eye and had impaired vision in the right eye that did not resolve after right eye surgery. The patient remained clinically stable over 5 years of followup and her last known CD4+ cell count was 479 cells/ $\mu \mathrm{L}$.

\section{Discussion}

Case 1 presented with mixed ocular pathology. HIV retinopathy presents in $70 \%$ of patients with advanced stage HIV disease ${ }^{[14]}$ and affects the posterior segment of the eye, as does CMV retinitis. HIV retinopathy is an occlusive microangiopathy, ${ }^{[15]}$ which presents as cotton wool spots, telangiectasia, microaneurysms and retinal haemorrhages. Cotton wool spots are the only common clinical feature in both HIV retinopathy and CMV retinitis; ${ }^{[16]}$ however, cotton wool spots associated with HIV retinopathy are usually superficial, smaller lesions that resolve within a few months. ${ }^{[16]}$ Immediate visual loss may not occur with HIV retinopathy and most often patients are asymptomatic. ${ }^{[16]}$ However, there may be significant damage to the retinal nerve fibre layer, deterioration in colour vision and visual field deficits. ${ }^{[6]}$ HIV retinopathy is a risk factor for CMV retinitis prompting the recommendation for routine ophthalmic examination every 3 months in patients with HIV retinopathy. ${ }^{[17]}$ It is hypothesised that the microvasculopathy associated with HIV retinopathy aids the transit of CMV-infected leukocytes across vessel walls. Data from the Longitudinal Studies of the Ocular Complications of AIDS (LSOCA) 
cohort $^{[4]}$ concluded that the presence of HIV retinopathy increased the risk of CMV retinitis (hazard ratio 11.6; 95\% CI 3.3 - 40.2; $p=0.0001$ ). It is important to note that other HIV-associated infectious causes of a retinitis such as varicella zoster virus, Toxoplasma gondii and choroidopathy caused by Pneumocystis carinii, should be excluded. ${ }^{[15]}$ Cryptococcal ocular complications can manifest in patients with advanced stage HIV disease who develop cryptococcal meningitis and subsequent optic neuritis and papilloedema. ${ }^{[18]}$ Ocular TB has been described in HIV-infected patients with disseminated TB affecting both the anterior and posterior segments of the eye with a variety of presentations that include: vitritis, retinal vasculitis, optic neuritis, choroiditis and choroidal tubercules. ${ }^{[6,19]}$ Retinal toxicity secondary to ARTs, and to drugs used to treat commonly occurring HIV-associated comorbidities such as tuberculosis therapy, has been described in the literature. Didanosine, which was one of the earliest nucleoside reverse transcriptase inhibitors, resulted in retinal toxicity in patients initiated on ART, ${ }^{[20]}$ and ritonavir- and efavirenzinduced retinal pigment eptheliopathy (RPE) initiating a 'bull's eye maculopathy' have also been reported in the literature. ${ }^{[21,22]}$

Owing to its cost-effectiveness, intravitreal ganciclovir injection is the current standard treatment in the SA public sector for active CMV retinitis infection, ${ }^{[23]}$ even though systemic ganciclovir or valganciclovir therapy is standard of care in other countries as these drugs assist in the prevention of systemic dissemination and improving survival. ${ }^{[3,23]}$ These injections are prescribed in combination with daily ART; the dose is based on clinical response and immune recovery and is stopped when CD4+ cell counts of $>100$ cells $/ \mu \mathrm{L}$ are achieved. ${ }^{[24]}$ The third case developed retinal detachment, which is a severe complication of CMV retinitis. Risk factors for retinal detachment include large anterior retinal lesions, intravitreal ganciclovir injections and bilateral disease. ${ }^{[25]}$ While our patient demonstrated bilateral disease, she did not receive intravitreal injections, and we were unable to comment on the size of the anterior retinal lesion owing to the extent of the pathology on presentation.

Retinal detachment is associated with poor visual outcomes leading to doubling of the visual angle and eventual blindness despite the initiation of ART, as was noted in our patient. ${ }^{[3,26]}$ This patient reported an onset of visual loss post ART initiation and could have been a case of missed CMV retinitis at screening as she was asymptomatic. An important consideration in this patient would be an immune reconstitution inflammatory syndrome presenting as an immune recovery vitritis or immune recovery uveitis (IRU). IRU is a paradoxical worsening of intraocular inflammatory reactions that occurs during immune recovery after the administration of ART and can lead to substantial visual loss. ${ }^{[23]}$ IRU can occur in $63 \%$ of patients with previous CMV retinitis ${ }^{[27]}$ and can present as a mild decrease in vision with the presence of floaters, pain and redness in the affected eye. ${ }^{[28]}$

Untreated CMV retinitis can lead to permanent blindness as a result of direct damage to the macula or optic nerve, or owing to retinal detachment. The need for surgical management to treat complications such as retinal detachment is often delayed in resource-limited settings and the persistent threat of developing CMV infection with chronic virological, immunological and clinical failure remains.

The three cases presented here highlight the severe and irreversible visual complications characteristic of CMV retinitis. Despite early identification and rapid referral to a tertiary ophthalmology service, all patients developed permanent and irreversible visual impairment. The greatest risk factor for active CMV retinitis infection is a CD4+ cell count of $<50$ cells $/ \mu \mathrm{L}^{[4,29,30]}$ in HIV infected patients, irrespective of whether patients are receiving ART. It is noteworthy that $90 \%$ of patients with incident CMV retinitis analysed in the LSOCA cohort $^{[31]}$ had a recent CD4+ cell count of $<50$ cells/ $\mu \mathrm{L}$, with $85 \%$ reporting ART use at the visit prior to CMV diagnosis. ${ }^{[4]}$

The mainstay of CMV retinitis management is disease prevention through early ART initiation, especially before CD4+ cell count levels decline to $<50$ cells $/ \mu \mathrm{L}$. Furthermore, the importance of conducting routine ophthalmological examinations on patients presenting for ART initiation with advanced disease needs to be emphasised. ${ }^{[2,31,32]}$ Visual loss adds to the overwhelming social and economic burden on patients and societies already plagued by HIV. We support the recommendation that routine fundoscopic examination be included in the standard WHO care package for HIV-infected patients with advanced disease. ${ }^{[10]}$ Similar to other opportunistic infections in HIV, CMV screening, prevention, early diagnosis and treatment access should also be offered to patients at high risk for this complication. While all our patients presented with a history of visual loss, published data from elsewhere show high rates of asymptomatic CMV retinitis. Data from Myanmar showed that $31 \%$ of the 42 patients diagnosed with CMV retinitis by fundoscopy, were asymptomatic. ${ }^{[2]}$

Asymptomatic CMV retinitis was also demonstrated in $44 \%$ of Cambodian patients, despite the use of a questionnaire designed specifically to elicit symptoms of CMV retinitis. ${ }^{[2]}$ While studies advocate education of patients with low CD4+ cell counts $(<100 \text { cells } / \mu \mathrm{L})^{[10]}$ on the risk of CMV retinitis, and highlight the need for urgent medical evaluation in patients presenting with ophthalmic symptoms, ${ }^{[33]}$ there are still large numbers of asymptomatic CMV retinitis cases. This underscores the need for the inclusion of routine fundoscopy in treatment guidelines for patients with advanced stage HIV as well as investment in training and infrastructure to enable frontline workers to conduct routine fundoscopic examinations on eligible patients. The training of nonophthalmologist clinicians in the use of telemedicine in screening for CMV retinitis, by means of digital fundus photography, should be explored..$^{[34]}$

\section{Study limitations}

This was a retrospective data review and there was missing information with regard to ophthalmology visits and the relevant feedback reports. In addition, images for two of the cases reviewed here could not be retrieved from the ophthalmology department owing to technical errors with equipment.

\section{Conclusion}

CMV retinitis is a preventable condition that has debilitating and permanent sequelae. Our review highlights the importance of ART and diagnosing patients in the early stages of HIV infection, as well as the importance of routine ophthalmoscopy in patients with advanced stage disease. CMV retinitis frequently affects young individuals in the most productive stage of life. Despite virological suppression and immune recovery on ART, vision cannot be restored and blindness leads to a life of dependency in such cases. ${ }^{[2]}$

CMV retinitis exists in the era of ART - we should not be lulled into believing otherwise. As we adopt the 'test and treat' strategy in SA, it is essential that frontline healthcare workers are trained in identifying CMV retinitis in high-risk individuals and that nonophthalmologist clinicians are equipped with the necessary skills to conduct ophthalmological examinations as part of routine HIV care. Furthermore, routine ophthalmological screening should be incorporated into standard treatment guidelines and algorithms for patients with $\mathrm{CD} 4+$ cell counts $<100$ cells $/ \mu \mathrm{L}$. 
Acknowledgements. The authors would like to thank the patients. Data were presented as a poster presentation at the 8th SA AIDS Conference held in Durban, SA on 13 June 2017.

Author contributions. RHM and KN wrote the initial draft of the manuscript. TC, LJ, LV and RHM assisted with data collection and interpretation. All authors participated in the writing and editing of the article.

Funding. Patients were enrolled into the CAT Programme, supported by the US President's Emergency Plan for AIDS Relief (PEPFAR), the Global Fund to fight AIDS, Tuberculosis and Malaria, and the National Institute of Health Comprehensive International Program of Research on AIDS. KN was supported by the Columbia University-South Africa Fogarty AIDS International Training and Research Program (Grant D43 TW000231).

Consent for publication. Written informed consent was obtained from the patients for publication of these case reviews and accompanying images. Conflicts of interest. KN received funding from PEPFAR, National Institutes of Health/NAID (Grant UM1AI069469) and Howard Hughes Medical Institute (Grant HHM1 55007065) for CAT. The other authors declare that they have no competing interests.

1. Butler NJ, Thorne JE. Current status of HIV infection and ocular disease. Curr Opin Ophthalmol 2012;23(6):517-522. https://doi.org/10.1097/ICU.0b013e328358ba85

2. Heiden D, Ford N, Wilson D, et al. Cytomegalovirus retinitis: The neglected disease of the AIDS pandemic. PLoS Med 2007;4(12):e334. https://doi.org/10.1371/journal.pmed.0040334

3. Jabs DA, Ahuja A, van Natta M, Dunn J, Yeh S. Comparison of treatment regimens for cytomegalovirus retinitis in patients with AIDS in the era of highly active antiretroviral therapy. Ophthalmology retinitis in patients with AIDS in the era of highly active antiretrovir

4. Sugar EA, Jabs DA, Ahuja A, et al. Incidence of cytomegalovirus retinitis in the era of highly active 4. Sugar EA, Jabs DA, Ahuja A, et al. Incidence of cytomegalovirus retinitis in the era of highly active
antiretroviral therapy. Am J Ophthalmol 2012;153(6):1016-1024.e5. https://doi.org/10.1016/j. ajo.2011.11.014

5. Martin-Odoom A, Bonney EY, Opoku DK. Ocular complications in HIV positive patients on antiretroviral therapy in Ghana. BMC Ophthalmol 2016;16(1):134. https://doi.org/10.1186/s12886 016-0310-5

6. Agarwal A, Singh R, Sharma A, Gupta V, Dogra MR. Ocular manifestations in patients with human immunodeficiency virus infection in the pre-HAART versus the HAART era in the North India population. Ocular Immunol Inflamm 2016:396-404. https://doi.org/10.3109/09273948.2015.1133837 7. Ford N, Shubber Z, Saranchuk P, et al. Burden of HIV-related cytomegalovirus retinitis in resourcelimited settings: A systematic review. Clin Infect Dis 2013;57(9):1351-1361. https://doi.org/10.1093/ $\mathrm{cid} / \mathrm{cit} 494$

8. Pathai S, Gilbert C, Weiss HA, McNally M, Lawn SD. Differing spectrum of HIV-associated ophthalmic disease among patients starting antiretroviral therapy in India and South Africa. Top Med Int Health disease among patients starting antiretroviral therapy in India and Sou

9. EL Fane M, Sodqi M, El Rherbi A, et al. Cytomegalovirus disease in patient with HIV infection. . EL Fane M, Sodqi M, El Rherbi A, et al. Cytomegalovirus disease in patien
J Antimicrob Agents 2016;2(1). https://doi.org/10.4172/2472-1212.1000108

10. Heiden D, Saranchuk P, Tun N, et al. We urge WHO to act on cytomegalovirus retinitis. Lancet Glob 10. Heiden D, Saranchuk P, Tun N, et al. We urge WHO to act on cytomegalo
Health 2014;2(2):e76-e77. https://doi.org/10.1016/S2214-109X(13)70174-8

11. Chiotan C, Radu L, Serban R, Cornăcel C, Cioboata M, Anghel A. Cytomegalovirus retinitis in HIV/ AIDS patients. J Med Life 2014;7(2):237.
12. Heiden D, Tun N, Maningding E, et al. Training clinicians treating HIV to diagnose cytomegalovirus retinitis. Bull World Health Org 2014;92(12):903-908. https://doi.org/10.2471/BLT.14.142372

13. Chakraborty D, Rama SK. CMV retinitis in an HIV patient with clinical and immunological Chakraborty D, Rama SK. CMV retinitis in an HIV patient with clinical and immunological
failure on HAART. Int J Med Science Pub Health 2015;4(1):142-144. https://doi.org/10.5455/ failure on HAART. In
ijmsph.2015.150820142

14. Jabs DA. AIDS retinopathy: Findings and significance. Ophthalmol Clin North Am 1988;1:101.

15. Jabs DA. Ocular manifestations of HIV infection. Trans Am Ophthalmol Soc 1995;93:623.

16. Holland GN. AIDS and ophthalmology: The first quarter century. Am J Ophthalmol 2008;145(3):397408.e391. https://doi.org/10.1016/j.ajo.2007.12.001

17. Nishijima T, Yashiro $\mathrm{S}$, Teruya K, et al. Routine eye screening by an ophthalmologist is clinically useful for HIV-1-infected patients with CD4 count less than 200/muL. PLoS One 2015;10(9):e0136747. https://doi.org/10.1371/journal.pone.0136747

18. Moodley A, Rae W, Bhigjee A. Visual loss in HIV-associated cryptococcal meningitis: A case series and review of the mechanisms involved. South Afr J HIV Med 2015;16(1):1-9. https://doi.org/10.4102/ sajhivmed.v16i1.305

19. Gupta V, Shoughy SS, Mahajan S, et al. Clinics of ocular tuberculosis. Ocul Immunol Inflamm 2015;23(1):14-24. https://doi.org/10.3109/09273948.2014.986582

20. Gabrielian A, MacCumber MM, Kukuyev A, Mitsuyasu R, Holland GN, Sarraf D. Didanosineassociated retinal toxicity in adults infected with human immunodeficiency virus. JAMA Ophthalmol 2013;131(2):255-259. https://doi.org/10.1001/jamaophthalmol.2013.579

21. Pinto R, Vila-Franca M, Afonso CO, Ornelas C, Santos L. Ritonavir and bull's eye maculopathy: Case report. GMS Opthalmol Cases 2013;3:Doc01. https://doi.org/10.3205/oc000011

22. Vaz Pereira C, Franco M, Guerra Pinto R, et al. Bull's eye maculopathy in a patient treated with efavirenz. Oftalmologia 2014;38(4):287-290.

23. Lapere SR, Rice JC. Cytomegalovirus retinitis in Cape Town, South Africa: Clinical management and outcomes. S Afr Med J 2017;107(6):514-517. https://doi.org/10.7196/samj.2017.v107i6.12250

24. Gore SK, Gore DM, Chetty K, Visser L. Cytomegaloviral retinitis-related retinal detachment: Outcomes following vitrectomy in the developing world. Int Opthalmol 2014;34(2):205-210. https:// doi.org/10.1007/s10792-013-9814-7

25. Yen M, Chen J, Ausayakhun S, et al. Retinal detachment associated with AIDS-related cytomegalovirus retinitis: Risk factors in a resource-limited setting. Am J Opthalmol 2015;159(1):185-192. https://doi. org/10.1016/j.ajo.2014.10.014

26. Thorne JE, Jabs DA, Kempen JH, et al. Incidence of and risk factors for visual acuity loss among patients with AIDS and cytomegalovirus retinitis in the era of highly active antiretroviral therapy. patients with AIDS and cytomegalovirus retinitis in the era of highly active antiretro

27. Urban B, Bakunowicz-Eazarczyk A, Michalczuk M. Immune recovery uveitis: Pathogenesis, clinical symptoms, and treatment. Mediators Inflamm 2014;2014(2014):Article ID 971417. https://doi. $\mathrm{rg} / 10.1155 / 2014 / 971417$

28. Balamurugan S, Khodifad A. Immune recovery uveitis. The Official Scientific J Delhi Ophthalmol Soc 2015;25(4):233-237. https://doi.org/10.7869/djo.114

29. Ausayakhun S, Keenan JD, Ausayakhun S, et al. Clinical features of newly diagnosed cytomegalovirus retinitis in northern Thailand. Am J Ophthalmol 2012;153(5):923-931. https://doi.org/10.1016/j. ajo. 2011.10 .012

30. Leenasirimakul P, Liu Y, Jirawison C, et al. Risk factors for CMV retinitis among individuals with HIV and low CD4 count in northern Thailand: Importance of access to healthcare. Br J Ophthalmol 2016;100:1017-1021. https://doi.org/10.1136/bjophthalmol-2016-308556

31. Jabs DA, van Natta ML, Holbrook JT, Kempen JH, Meinert CL, Davis MD. Longitudinal study of the ocular complications of AIDS: 1. Ocular diagnoses at enrollment. Ophthalmology 2007;114(4):780786. https://doi.org/10.1016/j.ophtha.2006.11.008

32. Tun N, Smithuis FM, London N, Drew WL, Heiden D. Mortality in patients with AIDS-related Tun N, Smithuis FM, London N, Drew WL, Heiden D. Mortality in patients with AIDS-related
cytomegalovirus retinitis in Myanmar. Clin Infect Dis 2014;59(11):1650. https://doi.org/10.1093/cid/ ciu648

33. Colby DJ, Vo DQ, Teoh SC, et al. Prevalence and predictors of cytomegalovirus retinitis in HIVinfected patients with low CD4 lymphocyte counts in Vietnam. Int J STD AIDS 2014;25(7):516-522. https://doi.org/10.1177/0956462413515197

34. Yen M, Ausayakhun S, Chen J, et al. Telemedicine diagnosis of cytomegalovirus retinitis by nonophthalmologists. JAMA Ophthalmol 2014;132(9):1052-1058. https://doi.org/10.1001/ jamaophthalmol.2014.1108

Accepted 25 August 2017. 